\title{
The association between retraction of the torn rotator cuff and increasing expression of hypoxia inducible factor $1 \alpha$ and vascular endothelial growth factor expression: an immunohistological study
}

Stefan Lakemeier*, Johannes JA Reichelt, Thilo Patzer, Susanne Fuchs-Winkelmann, Juergen RJ Paletta, Markus D Schofer

\begin{abstract}
Background: Differing levels of tendon retraction are found in full-thickness rotator cuff tears. The pathophysiology of tendon degeneration and retraction is unclear. Neoangiogenesis in tendon parenchyma indicates degeneration. Hypoxia inducible factor $1 \alpha$ (HIF) and vascular endothelial growth factor (VEGF) are important inducers of neoangiogenesis. Rotator cuff tendons rupture leads to fatty muscle infiltration (FI) and muscle atrophy (MA). The aim of this study is to clarify the relationship between HIF and VEGF expression, neoangiogenesis, $\mathrm{Fl}$, and MA in tendon retraction found in full-thickness rotator cuff tears.

Methods: Rotator cuff tendon samples of 33 patients with full-thickness medium-sized rotator cuff tears were harvested during reconstructive surgery. The samples were dehydrated and paraffin embedded. For immunohistological determination of VEGF and HIF expression, sample slices were strained with VEGF and HIF antibody dilution. Vessel density and vessel size were determined after Masson-Goldner staining of sample slices. The extent of tendon retraction was determined intraoperatively according to Patte's classification. Patients were assigned to 4 categories based upon Patte tendon retraction grade, including one control group. FI and MA were measured on standardized preoperative shoulder MRI.

Results: HIF and VEGF expression, Fl, and MA were significantly higher in torn cuff samples compared with healthy tissue $(p<0.05)$. HIF and VEGF expression, and vessel density significantly increased with extent of tendon retraction $(p<0.05)$. A correlation between HIFNEGF expression and FI and MA could be found $(p<0.05)$. There was no significant correlation between HIFNEGF expression and neovascularity $(p>0.05)$

Conclusion: Tendon retraction in full-thickness medium-sized rotator cuff tears is characterized by neovascularity, increased VEGF/HIF expression, FI, and MA. VEGF expression and neovascularity may be effective monitoring tools to assess tendon degeneration.
\end{abstract}

\section{Background}

Rotator cuff injury affects both the tendons and the associated muscles. Full thickness tears may result in muscle and tendon retraction, leading to a change in the angulation of muscle fibers and subsequent fatty

\footnotetext{
* Correspondence: lakemeie@med.uni-marburg.de

Department of Orthopedics and Rheumatology, University Hospital Marburg, Marburg, Germany
}

infiltration (FI) [1]. FI was first observed by computed tomography $(\mathrm{CT})$, which led to the five- stage1 classification system that is based upon fat-to-muscle ratio, one of the most important prognostic indicators after rotator cuff repair [1]. Furthermore, rotator cuff muscle atrophy (MA) is important in assessing treatment of rotator cuff tears [2-5]. Although these radiological parameters are good indicators of tendon degeneration, 
the histopathology of the degenerating rotator cuff are not as clear. Benson et al. proposed that hypoxic changes in the cuff contributes to loss of cells by apoptosis, which may be responsible for the development of tears [6]. These authors found different levels of hypoxia inducible factor $1 \alpha$ (HIF) in the damaged rotator cuff [7]. HIF is known to be an important upregulator of vascular endothelial growth factor (VEGF), which stimulates endothelial cells and vessels to invade hypovascularized tissue [8]. Pufe et al. and Peterson et al. found higher concentrations of VEGF in degenerative Achilles tendon as compared with healthy tissue $[9,10]$. Although the rotator cuff injury has been attributed to hypovascularity, there is some evidence that neovascularization also occurs [11]. Lewis et al. described increased neovascularity in patients with rotator cuff tendinopathy; increased HIF and VEGF expression in the diseased rotator cuff, but not in tendon retraction [12]. Furthermore, neovascularity has not been definitively proven histologically until this point.

The purpose of this study is to examine HIF and VEGF expression, and neovascularity in torn rotator cuff tissue from patients with medium size $(3-5 \mathrm{~cm}$, formally Bateman stage 3) full-thickness tears and tendon retraction, compared with healthy control samples. A second goal is to find an association between FI, MA and the amount of VEGF and HIF expression and neovascularity within both torn rotator cuffs.

\section{Methods}

\section{Patients}

39 patients (15 male, 24 female) was prospectively included in our study. Ethical approval was obtained from the ethics committee of the Medical Faculty at the University of Marburg, Germany. 33 patients had fullthickness rotator cuff tears which were refixtaed and closely repaired arthroscopically by double-row suture bridge technique performed by the senior author. Rotator cuff specimens were harvested from the margin of the tear site of the supraspinatus tendon during surgery. The control group consisted of six trauma patients with humeral head fractures; normal rotator cuff samples were harvested during open reduction and internal fixation from the supraspinatus tendon. Shoulder osteoarthritis was excluded by magnetic resonance imaging (MRI). Only patients with medium-sized full-thickness rotator cuff tears (tear size $3-5 \mathrm{~cm}$ ) were included into this study. Tendon retraction was defined based on intraoperative findings using Patte's classification (stage 1: proximal stump close to enthesis, or bony insertion; stage 2: proximal stump at humeral head; stage 3: proximal stump at glenoid) [13]. Patients were assigned to 4 categories according to Patte stage and including one control group.

\section{Radiographic Assessment}

All patients underwent preoperative shoulder MRI. FI was assessed according to the Goutallier-derived classification as adapted for use on oblique parasaggital T1-weighted fast-spin echo sequences $[1,5]$. The stages identified are: stage 0 , normal muscle; stage 1 , some fatty streaks; stage 2, manisfest fatty infiltration, but less than muscle; stage 3, as much fat asmuscle; and stage 4, more fat than muscle. FI was expressed for the supraspinatus muscle. Supraspinatus MA was measured on the most lateral image on which the scapular spine was in contact with the scapular body on oblique and saggital views as described by Thomazeau et al [4]. The crosssectional area of the supraspinatus muscle and its fossa were digitally measured using a picture analyzing system (PACS, Agfa-Gevaert group, Mortsel, Belgium). Results were expressed as the standadized muscle area, defined by the cross-sectional area of the muscle to the crosssectional area of the fossa $[5,14]$.

\section{Specimen Preparation}

Rotator cuff samples were immediately fixed in $4 \%$ formaldehyde for 24 hours, dehydrated in graded alcohol solution and cedarwood oil and embedded in paraffin. Sections were cut at $5 \mu \mathrm{m}$ with a Leica microtome RM2055 (Bensheim, Germany) 40 stainless-steel knife. Histological standard staining was performed with a Masson-Goldner staining kit (Merck, Dramstadt Germany) according to manufacturer instructions.

\section{Histology}

Histomorphometrical analysis was performed at a primary objective lens magnification of $5 \times$ using a Leica DM5000 and Quips analysis software (Leica Bensheim, Germany). In order to characterize the cells and to perform cell count, $40 \times$ objective lens magnification was used. For differential stained slices, for example Masson Goldner stained slices, a $10 \times$ objective lens magnification was used. Vessel number and size was determined by counting and measuring the vessels in three different areas of every specimen. Cell counting of immunohistological stained slices was performed by a $40 \times$ objective lens magnification. The percentage stands for the quotient of VEGF and HIF positive cells in relation to the total number of cells per slice. The percentage stands for the quotient of HIF or VEGF positive cells in relation to the total number of cells per slice. Figures 1, 2 and 3 show examples for different tendon sections and stainings.

\section{Immunochemistry}

For immunohistological staining of HIF and VEGF, the slices were rehydrated and incubated in citrate buffer $(\mathrm{pH} 6)$ at $90^{\circ} \mathrm{C}$ over a period of 10 minutes. After 

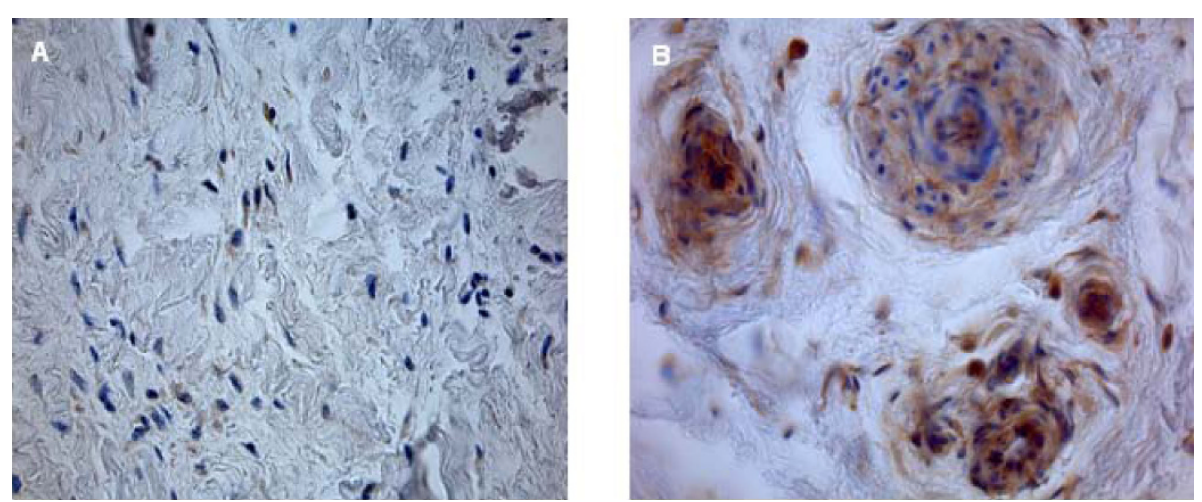

Figure 1 Rotator cuff slices after straining with VEGF antibody (40x magnification). In the control group, VEGF expression is minimally observed (a); VEGF expression is high in group IV (b).

blocking with normal horse serum, the slices were incubated overnight $\left(4^{\circ} \mathrm{C}\right.$, humidified chamber) with a monoclonal antibody: VEGF clone VG1; MAB3734, rabbit, dilution 1:750, Millipore, Bilerica, USA. HIF clone H1alpha67, mouse, dilution 1:100, Dianova, Hamburg, Germany.

Immunostaining was performed using the labeled streptavidin-biotin method (Dako REAL Detection System Peroxidase $/ \mathrm{DAB}+$ ), the staining reaction being based on 3,3'-diaminobenzidine (DAB). The stained slides were rinsed with distilled water and stained for 15 seconds with hematoxylin and eosin as a counterstain. Finally, the sections were rinsed with water and treated with graduated density alcohol and xylol.

\section{Statistics}

Analysis of variance (ANOVA) and a modified leastsquare difference (Bonferroni) test were used to evaluate the differences between the different groups. Data are reported as the mean \pm standard error of measurements
(SEM). Correlations between FI, MA, vessel count, VEGF and HIF were calculated according to Pearson's chi-square test. A p-value of less than 0.05 was considered significant.

\section{Results}

\section{Patient and Tissue Characteristics}

The supraspinatus muscle was injured in 100\% (33/33) of patients, the infraspinatus muscle in $55 \%(18 / 33)$. There were no subscapularis or teres minor tears. Patient demographics (age, gender), mean HIF and VEGF expression, vessel density, vessel size, and FI and MA classification schemes are outlined in Additional file 1.

\section{Changes in Cross-Sectional Muscle Area}

The fascial area was measured to be $212 \pm 114 \mathrm{~mm}^{2}$ in group I, $134 \pm 98 \mathrm{~mm}^{2}$ in group II, $142 \pm 87 \mathrm{~mm}^{2}$ in group III, and $120 \pm 58 \mathrm{~mm}^{2}$ in group IV. The bony area was $267 \pm 97 \mathrm{~mm}^{2}$ in group I, $245 \pm 87 \mathrm{~mm}^{2}$ in group II, $275 \pm 55 \mathrm{~mm}^{2}$ in group III, and $241 \pm 57 \mathrm{~mm}^{2}$
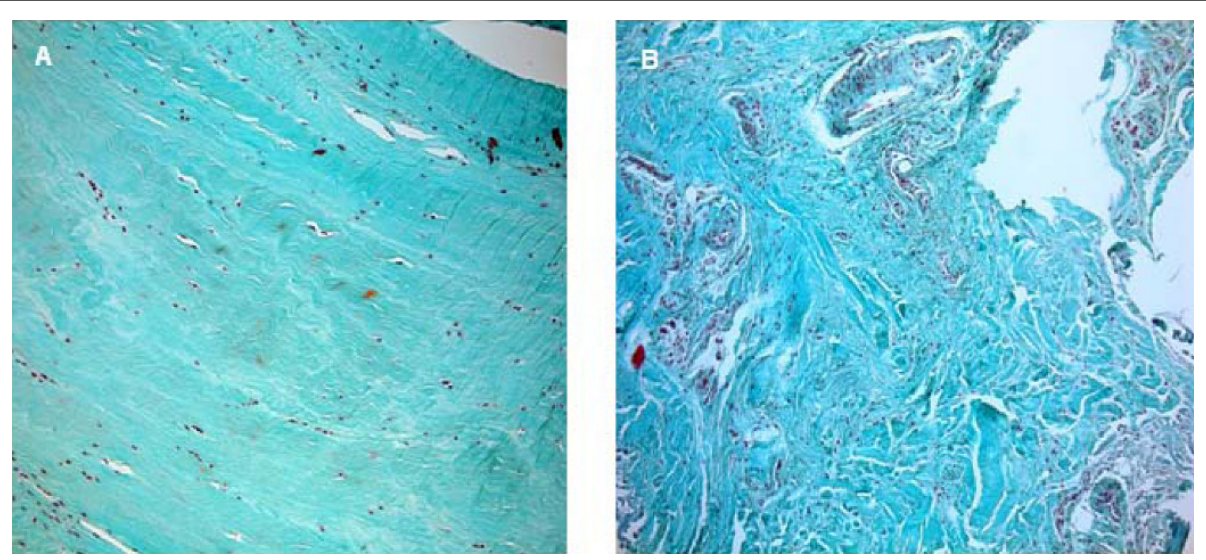

Figure 2 group II (Patte I) (a) has low vessel density. Group IV (Patte III) shows many visible vessels (b) (Rotator cuff slices, 10× magnification, Masson-Goldner staining). 

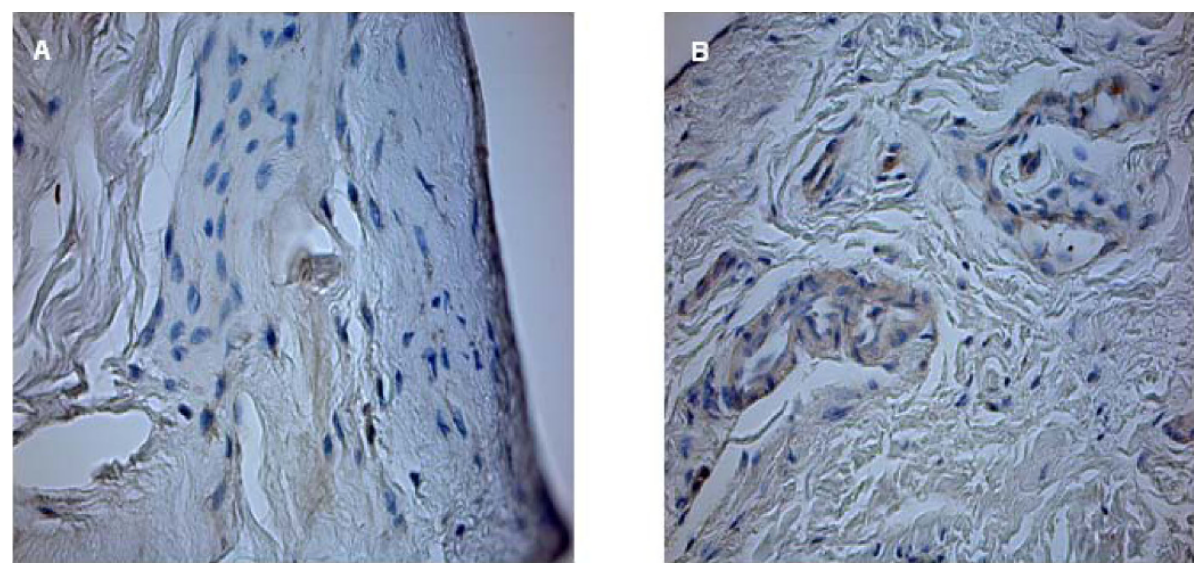

Figure 3 Rotator cuff slices after straining with HIF antibody (40x magnification). Minimal HIF expression is detected in group I (control group) (a). Many HIF positive cells in group IV (Patte III) (b).

in group IV. Differences between groups I and II-IV were significant on facial area $(p=0.031)$. Other differences between muscle areas were not statistically significant.

\section{Fatty Infiltration}

In the control group (group I), average FI was 0.5 (range $0-1$ ); in group II (Patte stage 1), 2.3 (range 1-3); in group III (Patte stage 2), 2.7 (range 2-4); and group IV (Patte stage 3) 3.5 (range 2-4). The differences in FI measurements between group I and II-IV were significant $(\mathrm{p}=$ 0.018 ) but not between groups II and III. FI was significantly higher in group IV (Patte stage 3 ) than in groups II and III (Patte stage 2-3) $(\mathrm{p}=0.036)$.

\section{HIF, VEGF Expression and Neovascularity}

HIF and VEGF expression was significantly elevated in groups II-IV (Patte stage 1-3) in comparison with controls (group I) $(p=0.045$, Figure 4a-d). Mean vessel density was significantly higher in group IV than in groups I, II and III $(\mathrm{p}=0.011)$. There was no correlation between vessel size and vessel density in groups II-IV compared with controls. Mean HIF and VEGF expression and vessel density is outlined in Additional file 1.

\section{Correlations}

We found a significant correlation between FI, MA, and HIF and VEGF expression in medium sized rotator cuff tears between groups II-IV and controls. There was a significant correlation between vessel density, FI, and fascial MA in group $4(\mathrm{p}=0.027)$. Other differences and correlations were not statistically significant.

\section{Discussion}

We aimed to clarity the relationship between VEGF/HIF expression and neovascularization and tendon retraction in full-thickness rotator cuff injuries. We showed that HIF and VEGF are upregulated in full-thickness rotator cuff tears (highest in group IV, Patte stage1 III), leading to higher vessel density while angiogenetic peptides are not detected in healthy tendon. In the torn rotator cuff, vessel density was lower than in our control group but increased with worsening tendon retraction. Our results confirm the findings of Matthews at al., who reported smaller numbers of vessels in massive tears compared to healthy controls [15]. However, vessel density was not explored in that work; for the first time, we have shown increasing vessel density correlating with retraction. This could be due to high VEGF levels, although we did not find this in our patient population. This may be due to the small number of patients in groups I and II.

While VEGF mediated angiogenesis contributes to the repair and remodeling of degenerated tendons, invasion of endothelial cells may also weaken the mechanical stability of the tendon [8]. We assume that increasing vessel density weakens the tendons and leads to the accretive nature of tendon retraction. This has been supported by the recently published theoretical model of Peers et al, showing that chronic tendon loading causes mechanical trauma with multiple microruptures of tendon microvasculature [16]. These microruptures initiate a VEGF-mediated cascade of vascular remodeling that becomes chronically pathologic. Hypervasularity has been demonstrated in degenerated human and rabbit Achilles tendons, in diseased patellar and long head biceps tendons [17-20]. Chronic tendon pathology appears to be a highly active process of ongoing neovascularisation [21]. Why this process does not lead to tissue repair, but to pain and chronic disease, is unknown.

Primary reconstruction of massive rotator cuff tears with minimal retraction is currently recommended in clinical practice; more extensive reconstruction with 


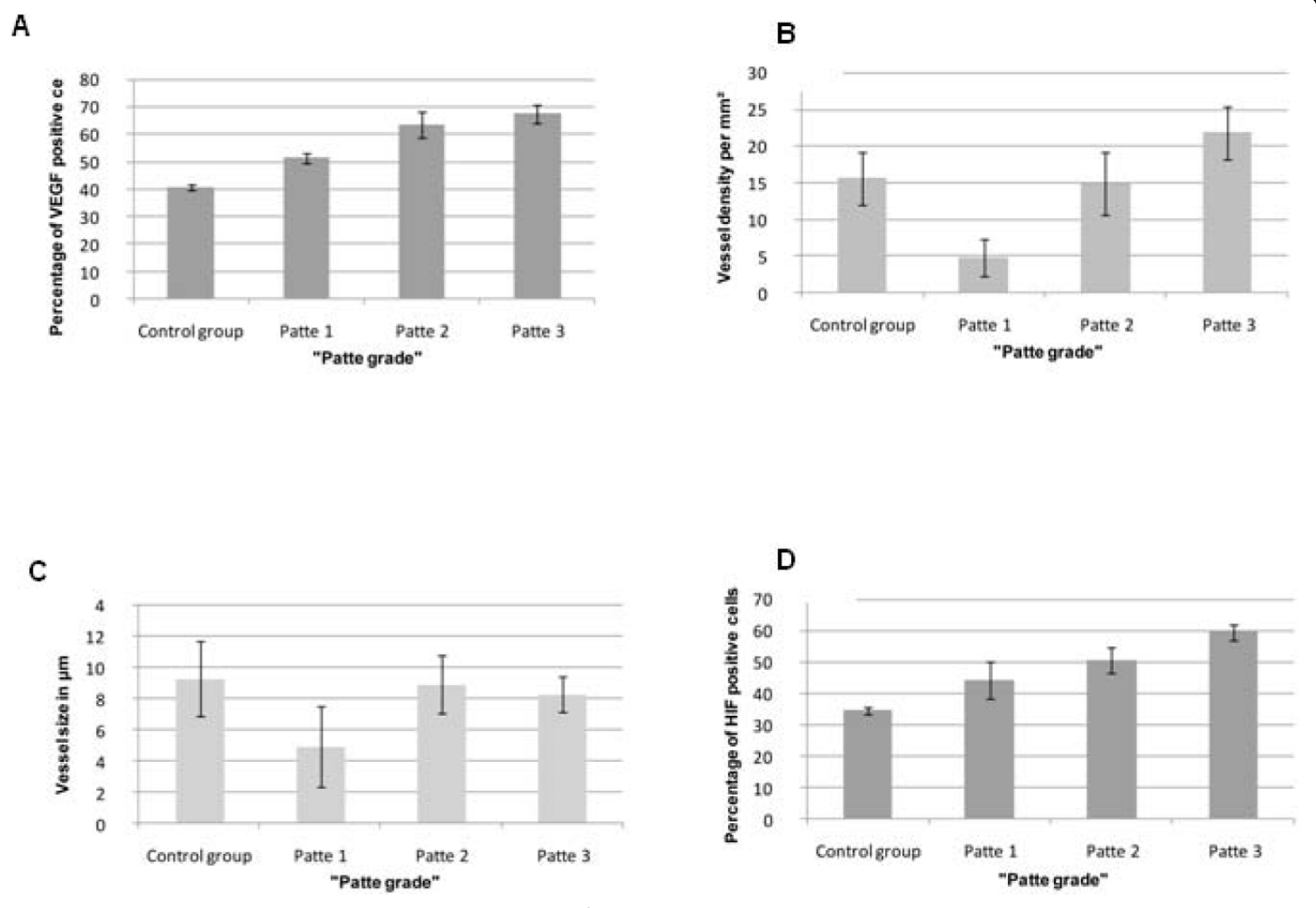

Figure 4 VEGF expression (a), vessel density (vessels $/ \mathrm{mm}^{2}$ ) (b), vessel size $(\mu \mathrm{m})(\mathrm{c})$ and percentage of HIF expression (d) for the different groups.

muscle transfers and prostheses are used in cases of moderate to severe retraction [22-24]. Our findings present histological backing for these guidelines and imply a potential benefit to treating patients with partial thickness tears more aggressively, in order to prevent complications of injury progression.

FI and MA of the torn rotator cuff correlate with the duration and size of the tear $[3,4,25]$. MA and FI levels could be used as outcome predictors for surgically treated injuries [26]. There is accumulating evidence that these parameters correlate closely with tendon degeneration. While age has been previously shown to correlate with FI and MA, we did not confirm this in our study. This may be due to the consistency of age among our patients groups. We demonstrated a correlation between FI and MA and VEGF/HIF expression. Therefore, VEGF and HIF expression as well as neovascularity may be used as a monitoring tool in the clinical setting, for the level of tendon degeneration that occurs during tendon degeneration. Neovascularization has been previously described in massive rotator cuff tears as compared to smaller tears of smaller size [27]. Neovascularization and lipid accumulation may be an indication of enhanced aerobic metabolism in the degenerated muscle. Further histological studies are required to investigate the mechanism of this finding; HIF and VEGF upregulation may help to explain these observations.

HIF, which is induced by cell hypoxia, is one of the most important regulators of VEGF gene expression [28]. The original function of VEGF as a strong inducer of embryonic vasculogenesis was shown in knockout mice [29]. VEGF plays a pivotal role in tumor angiogenesis (e.g. glioblastomas), as well as other disease states (diabetic retinopathy, age-related macular degeneration or rheumatoid arthritis) [28]. While the role of VEGF in tendon degeneration is largely unknown, the process is thought to occur in areas of poor perfusion. Rahburn and Macnab identified a hypovascularized zone in the rotator cuff that coincides with the most common location for tears [30]. In contrast, we found hypervascularisation within our samples. Our findings are supported by studies showing increased Doppler flow in diseased rotator cuffs, likely reflecting a neovascularization process $[12,31]$. 
The etiology of rotator cuff tears is still a matter of debate. Some authors ascribe mechanical damage to subacromial impingement, while others suspect a primary process of tendon degeneration [11]. On the basis of ultrasound findings, Gohlke found that patients with degenerative full-thickness rotator cuff tears and significant retraction were older [32]. In a recent literature review, Nho et al. conclude that hypovascularity is a minimal contributor to cuff tears, which supports our finding that hypervascularisation occurs late in the course of cuff tendinopathy [33]. Since HIF induction was initially described in conditions of decreased oxygen partial pressure, the majority of studies on HIF regulation have addressed hypoxic conditions [34]. However, there is evidence that HIF can also be induced under normal conditions by growth factors, hormones or nitric oxide [35].

Cyclic loading is as important inducer of HIF expression. Benson et al. showed HIF levels to be high in mild impingement, small, medium, and large tears, but reduced in massive tears. We assume that complete tendon rupture reduces mechanical cyclic loading, resulting in reduced HIF expression. If hypoxia underlies HIF expression, this contradicts Matthews's results describing the poorest blood supply in massive tears [15]. Biomechanical studies of Andarawis-Puri and Perry found out that cyclic tendon loading attenuates as retraction increases [36,37]. In our findings, HIF expression significantly increases with rising Patte grade. Therefore, it remains unclear whether hypoxia or cyclic tendon loading is the underlying reason for HIF and VEGF expression.

Our study has several limitations. Our control group is comparatively small; tumor patients were excluded from the study due to their requirement for prosthesis implantation or upper limb amputation. Therefore, we elected to include trauma patients of matchable age as controls (difficult due to the age discrepancy in this population as compared with the other groups and the reason for our low numbers).

Full-thickness rotator cuff tears may develop into cuff arthropathy and be associated with osteoarthritis of the shoulder [38]. Osteoarthritis was not measured, but may act as a confounder for VEGF and HIF expression. This bias was addressed by limiting our subjects to patients with medium sized tears $(3-5 \mathrm{~cm}$; unclassified due to lack of international consensus). Extent of tendon retraction was the only fluctuating parameter.

\section{Conclusion}

We demonstrated a correlation between vessel density, VEGF, and HIF expression as well as FI and MA in fullthickness medium-sized rotator cuff tears and tendon retraction. Neovascularization in cuff and tendon tissues is mediated by VEGF. Increasing vessel density accelerates tendon degeneration by weakening its underlying structure. VEGF expression and neovascularity could be used in the clinical setting to monitor tendon degeneration in patients with rotator cuff injuries.

\section{Additional material}

Additional file 1: Patient demographics with cuff tendon retraction classification, mean values for vessel density, vessel size, mean HIF and VEGF expression.

\section{Acknowledgements}

The authors thank the Kempkes-Foundation of Marburg, Germany for financially supporting this study. We thank Eleni Archontidou-Aprin and Soeren Schwuchow for supporting JJAR and JRJP in performing the histological analyses.

\section{Authors' contributions}

SL was the main composer of the manuscript; JJAR and JRJP performed histological and immunohistological testing. JRJP performed the statistical analysis and together with SL the radiographic assessments. SFW and TP conceived of the study, participated in its design and coordination, and helped to draft the manuscript. MDS designed the study, performed the surgeries, and obtained the tendon samples. All authors read and approved the final version of the manuscript.

\section{Competing interests}

The authors declare that they have no competing interests.

Received: 17 April 2010 Accepted: 8 October 2010

Published: 8 October 2010

\section{References}

1. Goutallier D, Bernageau J, Patte D: Assessment of the trophicity of the muscles of the ruptured rotator cuff by $C T$ scan. In Surgery of the shoulder. Edited by: Post M, Morrey B, Hawkins R. St. Louis: Mosby; 1990:11-13.

2. Mellado JM, Calmet J, Olona M, Esteve C, Camins A, Perez Del Palomar L, Gine J, Sauri A: Surgically repaired massive rotator cuff tears: MRI of tendon integrity, muscle fatty degeneration, and muscle atrophy correlated with intraoperative and clinical findings. AJR Am J Roentgenol 2005, 184(5):1456-1463.

3. Nakagaki K, Ozaki J, Tomita Y, Tamai S: Function of supraspinatus muscle with torn cuff evaluated by magnetic resonance imaging. Clin Orthop Relat Res 1995, , 318: 144-151.

4. Thomazeau H, Rolland Y, Lucas C, Duval JM, Langlais F: Atrophy of the supraspinatus belly. Assessment by MRI in 55 patients with rotator cuff pathology. Acta Orthop Scand 1996, 67(3):264-268

5. Zanetti M, Gerber C, Hodler J: Quantitative assessment of the muscles of the rotator cuff with magnetic resonance imaging. Invest Radiol 1998, 33(3):163-170.

6. Benson RT, McDonnell SM, Knowles HJ, Rees JL, Carr AJ, Hulley PA: Tendinopathy and tears of the rotator cuff are associated with hypoxia and apoptosis. J Bone Joint Surg Br 92(3):448-453.

7. Gerber HP, Condorelli F, Park J, Ferrara N: Differential transcriptional regulation of the two vascular endothelial growth factor receptor genes. Flt-1, but not Flk-1/KDR, is up-regulated by hypoxia. J Biol Chem 1997, 272(38):23659-23667.

8. Petersen W, Unterhauser F, Pufe T, Zantop T, Sudkamp NP, Weiler A: The angiogenic peptide vascular endothelial growth factor (VEGF) is expressed during the remodeling of free tendon grafts in sheep. Arch Orthop Trauma Surg 2003, 123(4):168-174.

9. Petersen W, Pufe T, Kurz B, Mentlein R, Tillmann B: Angiogenesis in fetal tendon development: spatial and temporal expression of the angiogenic 
peptide vascular endothelial cell growth factor. Anat Embryol (Berl) 2002, 205(4):263-270.

10. Pufe T, Petersen W, Tillmann B, Mentlein R: The angiogenic peptide vascular endothelial growth factor is expressed in foetal and ruptured tendons. Virchows Arch 2001, 439(4):579-585.

11. Lohr JF, Uhthoff HK: The microvascular pattern of the supraspinatus tendon. Clin Orthop Relat Res 1990, , 254: 35-38.

12. Lewis JS, Raza SA, Pilcher J, Heron C, Poloniecki JD: The prevalence of neovascularity in patients clinically diagnosed with rotator cuff tendinopathy. BMC Musculoskelet Disord 2009, 10:163.

13. Patte D: Classification of rotator cuff lesions. Clin Orthop Relat Res 1990, 254: 81-86.

14. Gerber C, Meyer DC, Frey E, von Rechenberg B, Hoppeler H, Frigg R, Jost B, Zumstein MA: Neer Award 2007: Reversion of structural muscle changes caused by chronic rotator cuff tears using continuous musculotendinous traction. An experimental study in sheep. J Shoulder Elbow Surg 2009, 18(2):163-171.

15. Matthews TJ, Hand GC, Rees JL, Athanasou NA, Carr AJ: Pathology of the torn rotator cuff tendon. Reduction in potential for repair as tear size increases. J Bone Joint Surg Br 2006, 88(4):489-495.

16. Peers KH, Brys PP, Lysens RJ: Correlation between power Doppler ultrasonography and clinical severity in Achilles tendinopathy. Int Orthop 2003, 27(3):180-183.

17. Backman C, Friden J, Widmark A: Blood flow in chronic Achilles tendinosis. Radioactive microsphere study in rabbits. Acta Orthop Scand 1991, 62(4):386-387.

18. Astrom M, Rausing A: Chronic Achilles tendinopathy. A survey of surgical and histopathologic findings. Clin Orthop Relat Res 1995, , 316: 151-164.

19. Khan KM, Bonar F, Desmond PM, Cook JL, Young DA, Visentini PJ, Fehrmann MW, Kiss ZS, O'Brien PA, Harcourt PR, et al: Patellar tendinosis (jumper's knee): findings at histopathologic examination, US, and MR imaging. Victorian Institute of Sport Tendon Study group. Radiology 1996, 200(3):821-827.

20. Lakemeier S, Reichelt JJ, Timmesfeld N, Fuchs-Winkelmann S, Paletta JR, Schofer MD: The relevance of long head biceps degeneration in the presence of rotator cuff tears. BMC Musculoskelet Disord 11:191.

21. Fenwick SA, Hazleman BL, Riley GP: The vasculature and its role in the damaged and healing tendon. Arthritis Res 2002, 4(4):252-260.

22. Kasten P, Loew M: [How to treat massive rotator cuff tears]. Orthopade 2007, 36(9):855-861.

23. Nho SJ, Delos D, Yadav H, Pensak M, Romeo AA, Warren RF, MacGillivray JD: Biomechanical and biologic augmentation for the treatment of massive rotator cuff tears. Am J Sports Med 38(3):619-629.

24. Schofer MD, Quante M, Peterlein CD, Timmesfeld N, Phan K, FuchsWinkelmann S: [Operative and follow-up treatment of rotator cuff tearsthe current situation]. Z Orthop Unfall 2009, 147(3):321-326.

25. Burke WS, Vangsness $C T$, Powers CM: Strengthening the supraspinatus: a clinical and biomechanical review. Clin Orthop Relat Res 2002, 402: 292-298.

26. Gladstone JN, Bishop JY, Lo IK, Flatow EL: Fatty infiltration and atrophy of the rotator cuff do not improve after rotator cuff repair and correlate with poor functional outcome. Am J Sports Med 2007, 35(5):719-728.

27. Steinbacher $P$, Tauber M, Kogler S, Stoiber W, Resch H, Sanger AM: Effects of rotator cuff ruptures on the cellular and intracellular composition of the human supraspinatus muscle. Tissue Cell 42(1):37-41.

28. Ferrara $\mathrm{N}$ : Vascular endothelial growth factor and the regulation of angiogenesis. Recent Prog Horm Res 2000, 55:15-35, discussion 35-16.

29. Giles PB, Candy CL, Fleming PA, Owens RW, Argraves WS, Drake CJ: VEGF directs newly gastrulated mesoderm to the endothelial lineage. Dev Biol 2005, 279(1):169-178.

30. Rathbun JB, Macnab I: The microvascular pattern of the rotator cuff. $J$ Bone Joint Surg Br 1970, 52(3):540-553.

31. Swiontkowski M, lanotti J, Boulas H, Esterhai J: Intraoperative assessment of rotator cuff vascularity using laser Doppler flowmetry. In Surgery of the shoulder. Edited by: Post M, Morrey B, RJ H. St. Louis: Mosby; 1990

32. Gohlke F: [Ultrasonographic appearance of the rotator cuff in elderly subjects]. Orthopade 1993, 22(5):288-293.

33. Nho SJ, Yadav H, Shindle MK, Macgillivray JD: Rotator cuff degeneration: etiology and pathogenesis. Am J Sports Med 2008, 36(5):987-993.

34. Wenger RH, Gassmann M: Oxygen(es) and the hypoxia-inducible factor-1. Biol Chem 1997, 378(7):609-616.
35. Kim WJ, Cho H, Lee SW, Kim YJ, Kim KW: Antisense-thioredoxin inhibits angiogenesis via $\mathrm{pVHL}$-mediated hypoxia-inducible factor-1alpha degradation. Int J Oncol 2005, 26(4):1049-1052.

36. Andarawis-Puri N, Ricchetti ET, Soslowsky L: Rotator cuff tendon strain correlates with tear propagation. J Biomech 2009, 42(2):158-163.

37. Perry $S M$, Getz CL, Soslowsky LJ: After rotator cuff tears, the remaining (intact) tendons are mechanically altered. J Shoulder Elbow Surg 2009, 18(1):52-57.

38. Pufe T, Lemke A, Kurz B, Petersen W, Tillmann B, Grodzinsky AJ, Mentlein R: Mechanical overload induces VEGF in cartilage discs via hypoxiainducible factor. Am J Pathol 2004, 164(1):185-192.

\section{Pre-publication history}

The pre-publication history for this paper can be accessed here: http://www.biomedcentral.com/1471-2474/11/230/prepub

\section{doi:10.1186/1471-2474-11-230}

Cite this article as: Lakemeier et al:: The association between retraction of the torn rotator cuff and increasing expression of hypoxia inducible factor $1 \alpha \alpha$ and vascular endothelial growth factor expression: an immunohistological study. BMC Musculoskeletal Disorders 2010 11:230.

\section{Submit your next manuscript to BioMed Central and take full advantage of:}

- Convenient online submission

- Thorough peer review

- No space constraints or color figure charges

- Immediate publication on acceptance

- Inclusion in PubMed, CAS, Scopus and Google Scholar

- Research which is freely available for redistribution

Submit your manuscript at www.biomedcentral.com/submit
C Biomed Central 